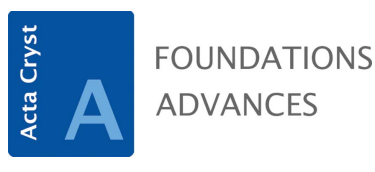

ISSN 2053-2733

Received 26 June 2017

Accepted 26 June 2017

Keywords: X-ray tomography; coherent diffraction imaging; biological imaging; contrasttransfer function

\section{Introducing the holo-TIE approach to cellular imaging}

\author{
Ian Robinson*
}

Condensed Matter Physics and Materials Science Division, Brookhaven National Laboratory, Upton, NY 11973-500, USA. *Correspondence e-mail: irobinson@bnl.gov

In this issue of Acta Crystallographica Section A, a significant advance in full-field X-ray imaging of biological samples is reported by workers from Tim Salditt's group at GeorgAugust-University in Göttingen (Krenkel et al., 2017). Their holographic regime method, which they call the holo-TIE approach, is a significant improvement of the classical 'transport of intensity' (TIE) method of Paganin \& Nugent (1998).

Following the original development of Cloetens et al. (1999), the work described by Krenkel et al. combines data measured at multiple detector distances to fill gaps of missing frequencies in the contrast-transfer function. This idea is presented and justified using the language of variation of Fresnel numbers.

The results also clearly show the respective advantages of different staining strategies relevant to biological imaging, where both resolution and contrast are key metrics. In $\mathrm{X}$-ray imaging science, 'resolution' is defined as the highest spatial frequency capable of being measured by the instrument, limited by the solid angle passed by some aperture or by the detector, for example. The 'contrast', on the other hand, is a property of the sample, measured in an analogous way to the presence of density (or phase) modulations of a given spatial frequency within the sample. Both the instrument and the sample have to deliver this level of performance in order for the resulting image to contain the fine features needed for biological interpretation. In the current work, reporting holo-TIE imaging of mouse macrophage cells (Krenkel et al., 2017), $\mathrm{OsO}_{4}$ was found to create a uniform background that identifies only the approximate shape of the cytoplasm-filled regions, while $\mathrm{BaSO}_{4}$ particles were found to stand out nicely. However, while the $\mathrm{BaSO}_{4}$ particles are seen with high resolution, they are markers that may or may not report on the biological state of the sample, depending on the method of attachment and introduction to the cells. Observing the markers with high resolution does not necessarily mean that the biological functions are determined with the same level of detail.

\section{Funding information}

Work at Brookhaven National Laboratory was supported by the US Department of Energy, Office of Science, Office of Basic Energy Sciences, under Contract DESC00112704.

\section{References}

Cloetens, P., Ludwig, W., Baruchel, J., Van Dyck, J., Van Landuyt, D., Guigay, J. \& Schlenker, M. (1999). Appl. Phys. Lett. 75, 2912-2914.

Krenkel, M., Toepperwien, M., Alves, F. \& Salditt, T. (2017). Acta Cryst. A73, 282-292. Paganin, D. \& Nugent, K. A. (1998). Phys. Rev. Lett. 80, 2586-2589. 